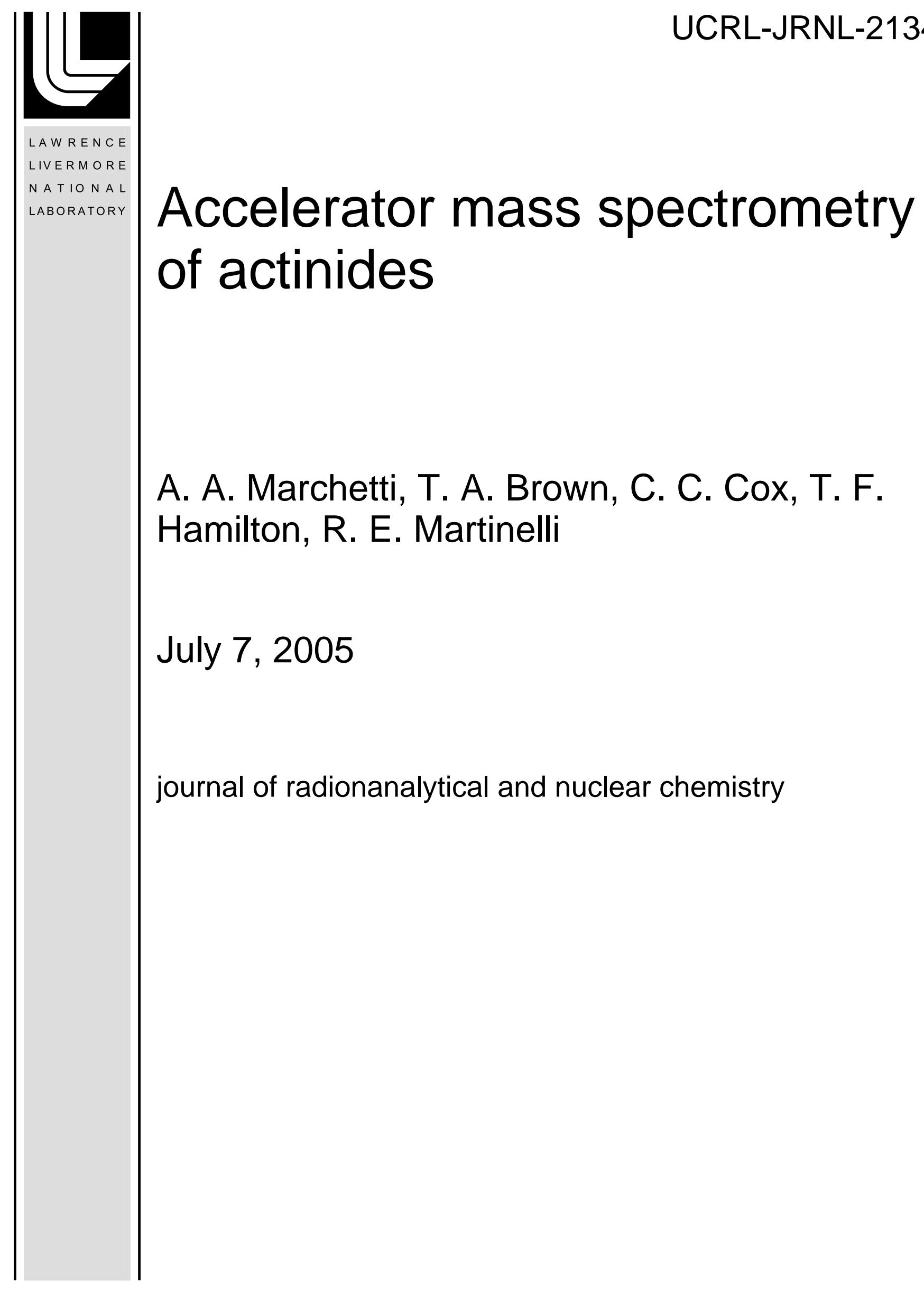


This document was prepared as an account of work sponsored by an agency of the United States Government. Neither the United States Government nor the University of California nor any of their employees, makes any warranty, express or implied, or assumes any legal liability or responsibility for the accuracy, completeness, or usefulness of any information, apparatus, product, or process disclosed, or represents that its use would not infringe privately owned rights. Reference herein to any specific commercial product, process, or service by trade name, trademark, manufacturer, or otherwise, does not necessarily constitute or imply its endorsement, recommendation, or favoring by the United States Government or the University of California. The views and opinions of authors expressed herein do not necessarily state or reflect those of the United States Government or the University of California, and shall not be used for advertising or product endorsement purposes. 
LOG NUMBER: 273

TITLE: ACCELERATOR MASS SPECTROMETRY OF ACTINIDES

AUTHORS: A.A. MARCHETTI, T.A. BROWN, C.C. COX, T.F.HAMILTON, R.E. MARTINELLI

ADDRESS: LAWRENCE LIVERMORE NATIONAL LABORATORY, 7000

EAST AVENUE, LIVERMORE, CA, 94550, USA

CORRESPONDING AUTHOR: ALFREDO MARCHETTI, FAX (925) 422-5673, E-MAIL MARCHETTI1@LLNL.GOV 


\title{
Accelerator mass spectrometry of actinides
}

\author{
A.A. Marchetti*, T.A. Brown, C. C. Cox, T.F. Hamilton, R.E. Martinelli \\ Lawrence Livermore National Laboratory, 7000 East Avenue, Livermore, CA \\ 94550, USA
}

Accelerator mass spectrometry (AMS) is a sensitive and robust technique typically applied to the quantification of long-lived radioisotopes in samples too small to be decay-counted. AMS is characterized by a high rejection of interferences and a low susceptibility to matrix components, which reduce the demands on sample preparation chemistry. At Lawrence Livermore National Laboratory (LLNL) Center for Accelerator Mass Spectrometry (CAMS), we have developed an AMS capability for the measurement of actinide concentrations and isotopic ratios. To date, this capability has been primarily devoted to the measurement of ${ }^{239} \mathrm{Pu}$ and ${ }^{240} \mathrm{Pu}$ in bioassay and environmental samples including soils, sediments, waters, and human urine. For these analyses, a known amount of ${ }^{242} \mathrm{Pu}$ is added to the samples as a reference isotope for normalization. Measurements of standard and intercomparison samples have shown that quantification is accurate and precise from at least $10^{6}$ to $10^{11}$ atoms/sample. Recently, the ratios of ${ }^{240} \mathrm{Pu},{ }^{241} \mathrm{Pu},{ }^{242} \mathrm{Pu}$, and ${ }^{244} \mathrm{Pu}$ to intrinsic ${ }^{239} \mathrm{Pu}$ have been successfully measured in soil samples from nuclear test sites. In addition, initial measurements of $\mathrm{U}$ and $\mathrm{Np}$ isotopes have yielded results consistent with the $\mathrm{Pu}$ measurements with respect to sensitivity, accuracy, precision, and linear range.

\footnotetext{
*E-mail: marchetti1@1lnl.gov
} 


\section{Introduction}

Measurements of plutonium isotopes and other actinides at very low concentrations are required in a number of important applications that include radiobioassay programs, environmental assessments, radioactive waste treatment, and basic research in chemistry and geochemistry. Atom counting techniques currently provide considerably better sensitivity than traditional radiometric methods for long-lived radionuclides. Among these techniques, AMS is widely used to measure very low levels of isotopes such as ${ }^{14} \mathrm{C},{ }^{10} \mathrm{Be},{ }^{26} \mathrm{Al},{ }^{36} \mathrm{Cl},{ }^{41} \mathrm{Ca}$, and ${ }^{129} \mathrm{I} .{ }^{1}$ In recent years, AMS has been further developed to incorporate the measurement of additional isotopes, particularly actinides, to its capabilities. ${ }^{2,3,4,5}$ Fifield et al. ${ }^{2}$ have demonstrated the feasibility of measuring plutonium isotopes using AMS with a limit of detection of $\sim 10^{6}$ atoms per sample, which is about two orders of magnitude lower than conventional counting by alpha spectrometry. AMS is also capable of distinguishing between ${ }^{239} \mathrm{Pu}$ and ${ }^{240} \mathrm{Pu}$, thus providing additional information that can be used for source identification. For example, the ratio ${ }^{240} \mathrm{Pu} /{ }^{239} \mathrm{Pu}$ due to integrated global fallout is on average 0.18 while that of weapons-grade $\mathrm{Pu}$ is $<0.07 .^{6}$ Other advantages of AMS are a large dynamic range, typically 5 orders of magnitude, and being less demanding on sample preparation than similarly sensitive techniques because of its high rejection of molecular isobaric interferences and low susceptibility to matrix effects.

An AMS system usually consists of a negative ion source, a low-energy spectrometer or injector system, a tandem accelerator, and a high-energy spectrometer. In general, the sample has to be in a solid form to be loaded into the ion source for measurement, e.g., graphite for ${ }^{14} \mathrm{C}$ measurements or $\mathrm{AgCl}$ for ${ }^{36} \mathrm{Cl}$ measurements. The 
sample is ionized by Cs sputtering and the negative ions produced are mass/energy analyzed and injected into the tandem accelerator where they are accelerated towards the positive terminal. At the positive terminal, the negative ions pass through a foil or gas stripper and lose electrons to become positive. During the stripping process, molecular ions are dissociated thereby reducing background interferences that may originate from isobaric hydrides or oxides. The positive ions are accelerated away from the positive terminal into the high-energy spectrometer, then mass/energy analyzed and, finally, counted in a gas ionization chamber or similar detector.

Due to the complexity of the processes involved in the ionization and transport of ions through the spectrometer, AMS requires that a reference isotope be measured concurrently with the isotope of interest for normalization. For example, ${ }^{14} \mathrm{C}$ is counted in an ionization chamber while stable ${ }^{13} \mathrm{C}$ or ${ }^{12} \mathrm{C}$ is measured as a current in a Faraday cup. The actual atomic ratio ${ }^{14} \mathrm{C} /{ }^{13} \mathrm{C}$ or ${ }^{14} \mathrm{C} /{ }^{12} \mathrm{C}$ is calibrated using standards. Normalization for actinides like plutonium is unique because there is no natural stable isotope. An isotope spike has to be added as reference and can also be used as a recovery tracer for quantification of the total atoms in the sample. In most practical actinide applications, the amount of reference isotope would be too low to be measured as a current using a Faraday cup. Therefore, the reference isotope must be counted as well. In some applications, it may be possible to choose one of the isotopes present in the sample as reference to obtain intrinsic ratios.

CAMS has recently added the capability of measuring plutonium and other actinides with the implementation of a heavy-ion beam line. ${ }^{7,8}$ Here we describe the CAMS heavy-ion spectrometer and its current measuring capabilities for actinides. 


\section{Experimental}

\section{Sample Preparation}

Actinide samples have to be converted to a manageable amount of solid to be put into the AMS ion source. In the case of environmental or bioassay samples, the actinides have to be solubilized by acid digestion, separated, and purified, following standard procedures common to other techniques such as alpha spectrometry. The purified actinide solution was taken to dryness, the actinide redissolved in $5 \mathrm{~mL}$ of $10 \mathrm{M} \mathrm{HCl}$, and coprecipitated with $0.15 \mathrm{mg}$ of $\mathrm{Fe}(\mathrm{III})$ by adding a $1: 1$ solution of $\mathrm{NH}_{4} \mathrm{OH}$. The precipitate was centrifuged, washed, redissolved in $0.15 \mathrm{~mL}$ of an $8 \mathrm{M} \mathrm{HNO}_{3}-15 \% \mathrm{H}_{2} \mathrm{O}_{2}$ solution containing $0.15 \mathrm{mg}$ of $\mathrm{Nb}(\mathrm{V})$, and transferred to a $\sim 1-\mathrm{mL}$ capacity quartz crucible where it was taken to dryness. The solid in the crucible was subsequently heated to $800^{\circ} \mathrm{C}$ in a muffle furnace to convert it to the oxide form. The solid, comprised mostly of $\mathrm{Fe}_{2} \mathrm{O}_{3}$ and $\mathrm{Nb}_{2} \mathrm{O}_{5}$, was scraped from the crucible and loaded in an aluminum target holder. Blanks and standards were prepared in the same manner to turn them into solid form. For routine measurements of ${ }^{239} \mathrm{Pu}$ and ${ }^{240} \mathrm{Pu}$, a pure ${ }^{242} \mathrm{Pu}$ spike was added as reference tracer. For test measurements of uranium isotopes, pure ${ }^{233} \mathrm{U}$ was used. For lack of an adequate neptunium isotope spike, ${ }^{242} \mathrm{Pu}$ was used for the measurement of ${ }^{237} \mathrm{~Np}$. Blanks, calibration curves, and standard isotopic solutions were used to evaluate the capabilities of the AMS system and for quality control during routine measurements. Accelerator Mass Spectrometry

The AMS heavy-ion beam line is illustrated in Fig. 1. The Cs-sputtering ion source produced $40 \mathrm{keV}$ negative ions (e.g., ${ }^{239} \mathrm{Pu}^{16} \mathrm{O}^{-},{ }^{236} \mathrm{U}^{16} \mathrm{O}^{-}$) ions that were transported first to a $90^{\circ}$ energy-analyzing spherical electrostatic deflector, and then to a 
$90^{\circ}$ mass-analyzing double-focusing magnet to be injected in the tandem accelerator ${ }^{\dagger}$ operated at $+6.5 \mathrm{MV}$. At the high-voltage terminal of the tandem, the negative molecular ions passed through a gas stripper and were converted to a charge distribution of positive atomic ions (e.g., ${ }^{239} \mathrm{Pu}^{5+},{ }^{239} \mathrm{Pu}^{6+}$, etc). Electrostatic repulsion then accelerated the positive ions out of the tandem through a $30^{\circ}$ mass-analyzing magnet into the heavy-ion beam line. The heavy-ion beam line is equipped with fast-switching electrostatic deflector plates, an energy-analyzing $45^{\circ}$ cylindrical electrostatic deflector, and a twoanode longitudinal-field ionization detector. The electrostatic deflector plates were used in combination with the fast mass switching capability of the low-energy spectrometer for isotope mass selection and fast normalization, i.e., fast switching between an isotope of interest and the reference isotope during measurement in time intervals of hundreds of milliseconds. This improves the accuracy and precision of the ratio because the ion source output remains essentially constant between the short sampling intervals.

The target wheel of the CAMS facility accommodates up to 64 samples. In automatic operation, the system could measure up to 5 different isotopes consecutively, repeat or move to another sample according to programmable instructions. During each isotope measurement cycle, the system alternated the mass selected for counting between that of an isotope of interest and that of the reference using sampling intervals of 400 and $100 \mathrm{msec}$, respectively. The measurement time per isotope was normally 10 seconds and the set of up to 5 isotopes was repeated 3 times per cycle for 15 total measurements per cycle. This measurement cycle was repeated 4 times for each sample. One of the 5 isotopes was selected to be the reference isotope. This measurement should yield a ratio

\footnotetext{
${ }^{\dagger}$ High Voltage Engineering Corporation, Model FN Tandem Accelerator
} 
of 1 and is used to normalize the data for any differences between the two channels (electronics, manual gates, etc.). The high-energy spectrometer was set to select the 5+ charge state $\left(E_{\text {kinetic }}=39 \mathrm{MeV}\right)$ for detection. The signals from the detector were used to set a charge gate to count the 5+ events only, using an interactive two-dimensional plot of the energy signal of one anode of the detector versus the other. This gate was sufficient to reject neighboring $4+$ and lower charge-state ions that make it to the detector.

\section{Results and discussion}

The detection efficiency of plutonium isotopes, based on samples with known amounts of atoms, is estimated at about $5 \times 10^{-5}$, a value that corresponds to a combination of the efficiencies of sample preparation and AMS. Note that the final stage in sample preparation is the transfer of $\sim 0.3 \mathrm{mg}$ of solid containing the actinide from a quartz crucible to a target holder. This process cannot be strictly quantitative because the crucible may adsorb some of the actinide and it is very difficult to get $100 \%$ yield in the transfer of the solid. To date, hundreds of measurements of ${ }^{239} \mathrm{Pu}$ and ${ }^{240} \mathrm{Pu}$ have been performed on bioassay and environmental samples by adding typically $3.0 \times 10^{9}$ atoms of

${ }^{242} \mathrm{Pu}$ as the reference isotope. In all of these measurements, blanks for ${ }^{239} \mathrm{Pu}$ and ${ }^{240} \mathrm{Pu}$ have been consistently below $10^{6}$ atoms. For example, the weighted averages and standard deviation of 18 and 10 sample blanks taken for ${ }^{239} \mathrm{Pu}$ during two separate runs were $0.05 \pm 0.29$ and $0.02 \pm 0.26 \times 10^{6}$ atoms, respectively.

Calibration samples have been included in every AMS run to cover the range of concentrations expected in the samples. A recent calibration curve ranging from $5.0 \times 10^{5}$ to $1.0 \times 10^{7}$ atoms of ${ }^{239} \mathrm{Pu}$ is shown in Fig. 2. A weighted least-squares linear fit to the data gave a slope of $1.146 \pm 0.041$ and an intercept of $(0.087 \pm 0.038) \times 10^{6}$ atoms of ${ }^{239} \mathrm{Pu}$. 
The slope is greater than 1 , which could be due to a difference between the nominal and actual concentration of the ${ }^{239} \mathrm{Pu}$ or ${ }^{242} \mathrm{Pu}$ solutions used to prepare the samples. Routine plutonium measurements also include samples of NIST traceable isotopic standards CRM 128 (present day ratio ${ }^{239} \mathrm{Pu} /{ }^{242} \mathrm{Pu}=0.9989 \pm 0.0003$ ) and $\mathrm{CRM} 138$ (present day ratio $\left.{ }^{240} \mathrm{Pu} /{ }^{239} \mathrm{Pu}=0.0862 \pm 0.0001\right)$ to make corrections for mass bias if needed. Most of our isotopic standard measurements to date have been in agreement with the expected values within the uncertainty, e.g., in a recent AMS run, the weighted mean of 6 CRM 138 and 6 CRM 128 samples were ${ }^{240} \mathrm{Pu} /{ }^{239} \mathrm{Pu}=0.0859 \pm 0.0012$ and ${ }^{239} \mathrm{Pu} /{ }^{242} \mathrm{Pu}=0.9968 \pm 0.0091$, respectively. In addition, the measurement of the reference isotope $\left({ }^{242} \mathrm{Pu} /{ }^{242} \mathrm{Pu}\right)$ has also been consistently 1 , indicating that there are no significant differences between measuring and reference channels.

A particular concern in the measurement of ${ }^{239} \mathrm{Pu}$ is the presence of uranium in the sample matrix. Uranium can produce molecular isobaric interferences during ionization in mass spectrometric measurements (e.g., ${ }^{238} \mathrm{UH}^{+}$at mass ${ }^{239} \mathrm{Pu}^{+},{ }^{238} \mathrm{U}^{17} \mathrm{O}^{+}$or ${ }^{238} \mathrm{U}^{16} \mathrm{OH}^{+}$ at mass ${ }^{239} \mathrm{Pu}^{16} \mathrm{O}^{+}$; similar cases occur for negative ions) and, for some techniques, special reagents and a high degree of sample purification are needed to attain $10^{6}$-atom sensitivity. To assess the interference of ${ }^{238} \mathrm{U}$ in ${ }^{239} \mathrm{Pu}$ measurements, samples containing approximately $10^{13}{ }^{238} \mathrm{U}$ atoms have been routinely measured demonstrating a rejection factor better than $10^{7}$. Therefore, sample preparation does not require special reagents for our ${ }^{239} \mathrm{Pu}$ measurements by AMS.

For the initial assessment of CAMS capabilities for uranium isotopes, we set out to measure ${ }^{234} \mathrm{U},{ }^{235} \mathrm{U}$, and ${ }^{236} \mathrm{U}$. A constant spike level of $2 \times 10^{9}$ atoms of ${ }^{233} \mathrm{U}$ was used as reference. Calibration samples for ${ }^{236} \mathrm{U}$ were prepared ranging from about $10^{5}$ to $10^{8}$ 
atoms. A weighted least-squares linear fit to the data, plotted in Fig. 3, yielded a slope of $1.046 \pm 0.015$ and an intercept of $(0.050 \pm 0.027) \times 10^{6}$ atoms of ${ }^{236} \mathrm{U}$. The weighted average of 3 blanks resulted in $(0.000 \pm 0.075) \times 10^{6}$ atoms of ${ }^{236} \mathrm{U}$. Three samples containing $\sim 10^{13}$ atoms of natural uranium were measured and the ratio ${ }^{234} \mathrm{U} /{ }^{235} \mathrm{U}$ was calculated from the measured ratios ${ }^{234} \mathrm{U} /{ }^{233} \mathrm{U}$ and ${ }^{235} \mathrm{U} /{ }^{233} \mathrm{U}$. The weighted average of the ratio ${ }^{234} \mathrm{U} /{ }^{235} \mathrm{U}$ was $0.0075 \pm 0.0002$ in good agreement with the expected value for natural uranium of $0.0075 \pm 0.0007 .^{9}$

To evaluate the capability of the AMS system to measure ${ }^{237} \mathrm{~Np}$, calibration samples containing nominally $10^{6}$ to $10^{10}$ atoms of ${ }^{237} \mathrm{~Np}$ and $3.0 \times 10^{9}$ atoms of ${ }^{242} \mathrm{Pu}$ as the reference isotope were prepared and measured (see Fig. 4). A weighted linear leastsquares fit to the data resulted in a slope of $0.603 \pm 0.013$ and an intercept of $(0.39 \pm 0.28) \times 10^{6}$ atoms. The slope is significantly less than 1.0. This is not unexpected since the reference isotope was a different element and would behave differently in the ion source. Fifield et al. ${ }^{3}$ measured the relative negative-ion formation for the monoxide ion of some actinides with respect to $\mathrm{PuO}^{-}$and the result for $\mathrm{NpO}^{-} / \mathrm{PuO}^{-}$was $0.77 \pm 0.03$. This value is significantly higher than the slope of our data. However, Fifield et al. used a matrix of $\mathrm{Fe}_{2} \mathrm{O}_{3}$ in their work while we used a matrix of $\mathrm{Fe}_{2} \mathrm{O}_{3}$ and $\mathrm{Nb}_{2} \mathrm{O}_{5}$ that could affect the ionization process and explain, at least partly, the difference observed. The weighted average of 6 blanks is $(0.34 \pm 0.23) \times 10^{6}$ atoms. Correction for the slope factor would yield a background level of $(0.56 \pm 0.38) \times 10^{6}$ atoms of ${ }^{237} \mathrm{~Np}$.

Among the applications of this technology, one of the most important is the determination of $\mathrm{Pu}$ in urine to assess dose or exposure in humans. Therefore, we have participated in a blind interlaboratory exercise designed to evaluate the capability of 
various techniques to measure ${ }^{239} \mathrm{Pu}$ and ${ }^{240} \mathrm{Pu}$ in this matrix. The results of this intercomparison demonstrate that AMS is capable of accurate and precise measurements down to $\sim 10^{-6} \mathrm{~Bq}\left(\sim 10^{6}\right.$ atoms $) .{ }^{10}$ Recently, the ratios of ${ }^{240} \mathrm{Pu},{ }^{241} \mathrm{Pu},{ }^{242} \mathrm{Pu}$, and ${ }^{244} \mathrm{Pu}$ to intrinsic ${ }^{239} \mathrm{Pu}$ have been successfully measured in soil samples from nuclear test sites. ${ }^{11}$

\section{Conclusions}

We have described the determination of actinides concentrations and isotope ratios using AMS at LLNL. Results from standard and intercomparison samples have shown that quantification is precise and accurate down to $\sim 10^{6}$ atoms for plutonium. Initial studies using uranium isotopes have yielded similar results. Direct measurements of isotopic ratios of plutonium standards and natural uranium show no significant mass bias within the uncertainties. Background determinations for ${ }^{239} \mathrm{Pu}$ are equivalent to $\sim 10^{5}$ atoms and samples containing ${ }^{238} \mathrm{U}$ demonstrated that this system has a rejection factor of $10^{7}$ or better for ${ }^{239} \mathrm{Pu}$ measurements. Initial measurements of ${ }^{237} \mathrm{~Np}$ using a ${ }^{242} \mathrm{Pu}$ spike as reference have shown similar results albeit at a somewhat smaller sensitivity and larger background. Sample preparation did not require special reagents or facilities, which together with the high-throughput design of the CAMS instrument offers a sensitive and cost-effective method to measure actinides with rapid turn around of results.

\section{Acknowledgements}

This work was performed under the auspices of the U.S. Department of Energy by the University of California, Lawrence Livermore National Laboratory under contract W7405-Eng-48. 


\section{References}

${ }^{1}$ J.S. Vogel, K.W. Turteltaub, R. Finkel, D.E. Nelson, Anal. Chem., 67 (1995) 353A.

${ }^{2}$ L.K. Fifield, R.G. Cresswell, M.L. Di TAda, T.R. OPhel, J.P. Day, A.P. Clacher, S.J. King, N.D. PRIEST, Nucl. Instr. and Meth. B, 117 (1996) 295.

${ }^{3}$ L.K. Fifield, A.P. Clacher, K. Morris, S.J. King, R.G. Cresswell, J.P. Day, F.R. Livens, Nucl. Instr. and Meth. B, 123 (1997) 400.

${ }^{4}$ X.-L. Zaho, L.R. Kilius, A.E. LitherLand, T.Beasley, Nucl. Instr. and Meth. B, 126 (1997) 297.

${ }^{5}$ C. Vochenhuber, I. Ahmad, R. Golser, W. Kutschera, V. Liechtesnstein, A. Priller, P. Steier, S. WINKLER, Int. J. Mass Spec. 223-224 (2003) 713.

${ }^{6}$ R.N. Taylor, T. Warneke, J.A. Milton, I.W. Croudace, P.E. Warwick, J. Anal. At. Spectrom., 16 (2001) 279 .

${ }^{7}$ J.E. McAninch, T.F. Hamilton, T.A. Brown, T.A. Jokela, J.P. Knezovich, T.J. Ognibene, I.D. Proctor, M.L. Roberts, E. Sideras-Haddad, J.R. Southon, J.S. Vogel, Nucl. Instr. and Meth. B, 172 (2000) 711.

${ }^{8}$ T.A. Brown, A.A. Marchetti, R.E. Martinelli, C.C. CoX, J.P. Knezovich, T.F. Hamilton, Nucl. Instr. and Meth. B, (in press).

${ }^{9}$ Isotopic composition for natural uranium obtained from: Nuclear Wallet Cards $6{ }^{\text {th }}$ Edition (August 30, 2001), Nuclear data Center, Brookhaven National Laboratory, Upton, NY.

${ }^{10}$ D. McCurdy, Z. Lin, K.G.W. InN, R. Bell III, S. Wagner, D.W. Enfurd, T. Hamilton, T.A. Brown, A.A. MARChETTI, J.Radioanal. Nucl. Chem., (this proceedings).

${ }^{11}$ T. Hamilton, T.A. Brown, A.A. Marchetti, R.E. Martinelli, S. Kehl, J. BrunK, J.Radioanal. Nucl. Chem., (this proceedings). 


\section{Figure captions}

Fig. 1. Diagram of the actinide AMS system at LLNL.

Fig. 2. AMS calibration curve of ${ }^{239} \mathrm{Pu}$. The curve consists of 6 blanks and 5 quantity levels in triplicate, with $3.0 \times 10^{9}$ atoms of ${ }^{242} \mathrm{Pu}$ as the reference isotope. A weighted least-squares fit to the data follows:

${ }^{239} \mathrm{Pu}_{\text {meas }}=\left[(1.146 \pm 0.041) \times{ }^{239} \mathrm{Pu}_{\text {expected }}+(0.087 \pm 0.038)\right] \times 10^{6}$ atoms, $\mathrm{r}^{2}=0.975$.

Fig. 3. AMS calibration curve of ${ }^{236} U$. The curve consists of 3 blanks (not shown) and 4 quantity levels in triplicate, with $2.0 \times 10^{9}$ atoms of ${ }^{233} \mathrm{U}$ as the reference isotope. A weighted least-squares fit to the data follows:

${ }^{236} \mathrm{U}_{\text {meas }}=\left[(1.046 \pm 0.015) \times{ }^{236} \mathrm{U}_{\text {expected }}+(0.050 \pm 0.027)\right] \times 10^{6}$ atoms, $\mathrm{r}^{2}=0.998$.

Fig. 4. AMS calibration curve of ${ }^{237} \mathrm{~Np}$. The curve consists of 6 blanks (not shown) and 5 quantity levels in triplicate, with $3.0 \times 10^{9}$ atoms of ${ }^{242} \mathrm{Pu}$ as the reference isotope. A weighted least-squares fit to the data follows:

${ }^{237} \mathrm{~N} p_{\text {meas }}=\left[(0.603 \pm 0.013) \times{ }^{237} \mathrm{~Np}_{\text {expected }}+(0.39 \pm 0.28)\right] \times 10^{6}$ atoms, $\mathrm{r}^{2}=0.990$. 
Fig. 1.

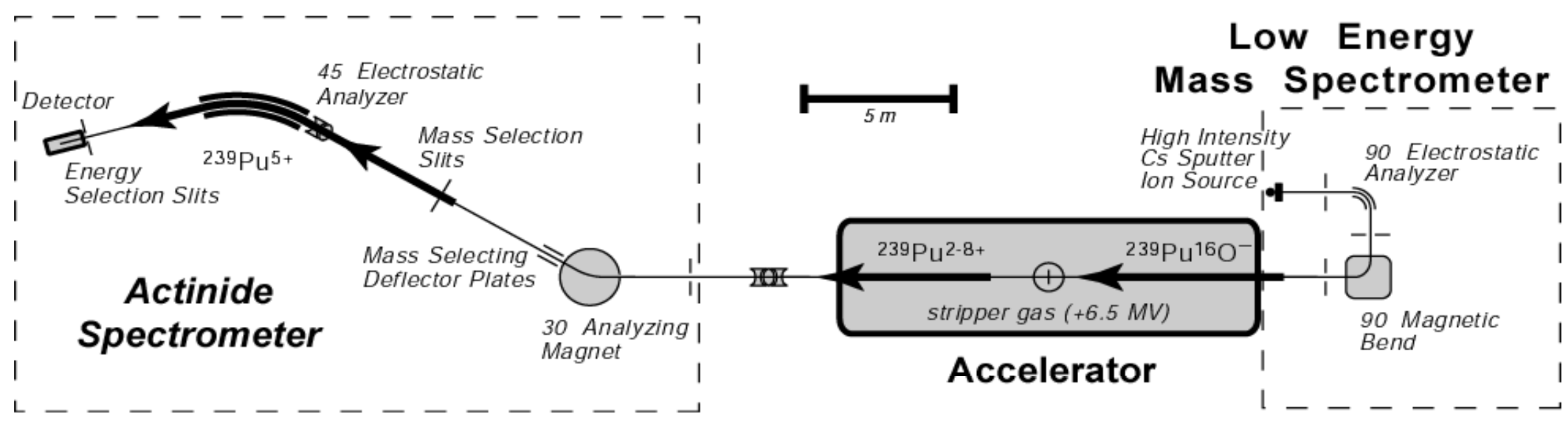




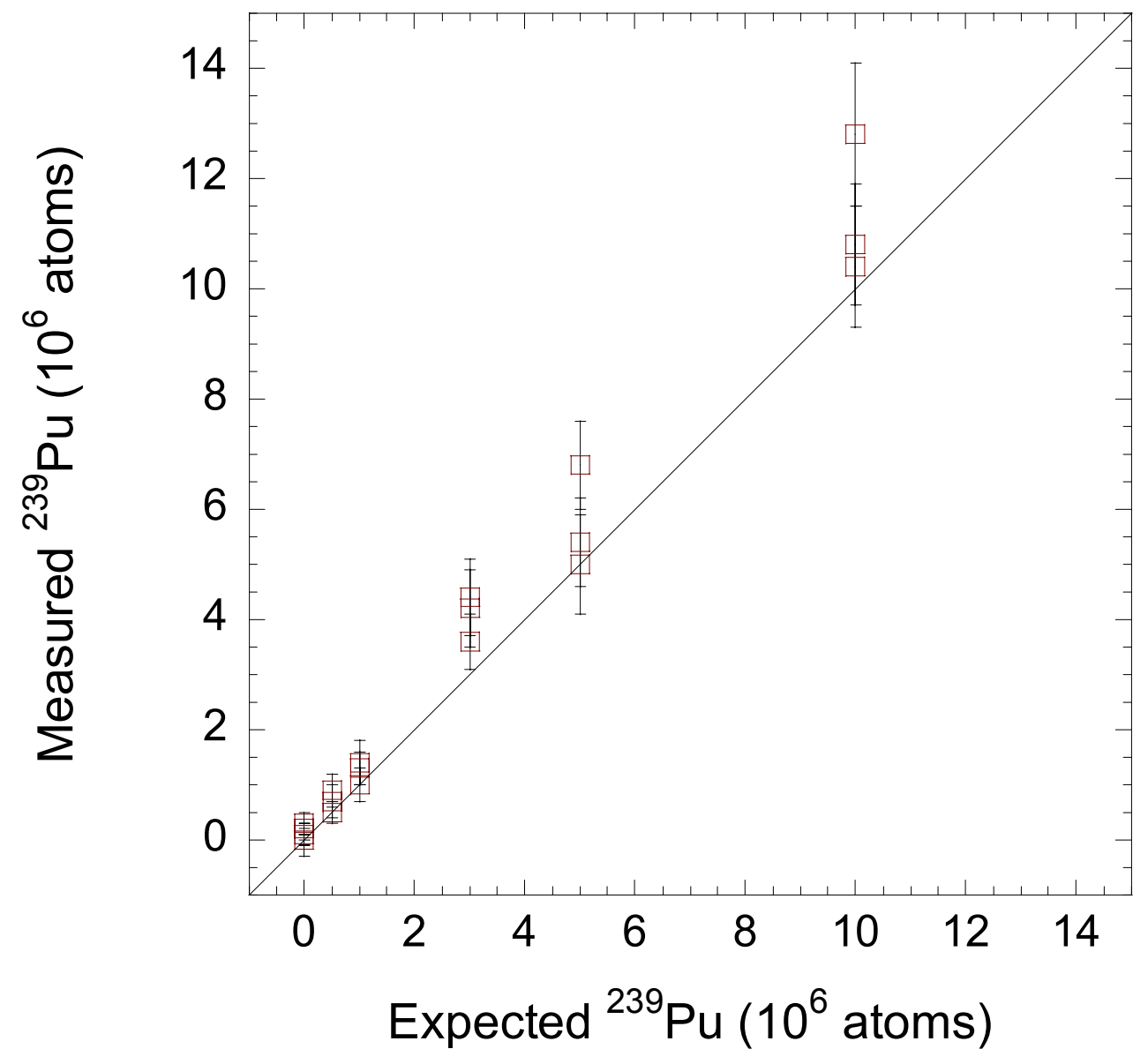

Fig. 2. 


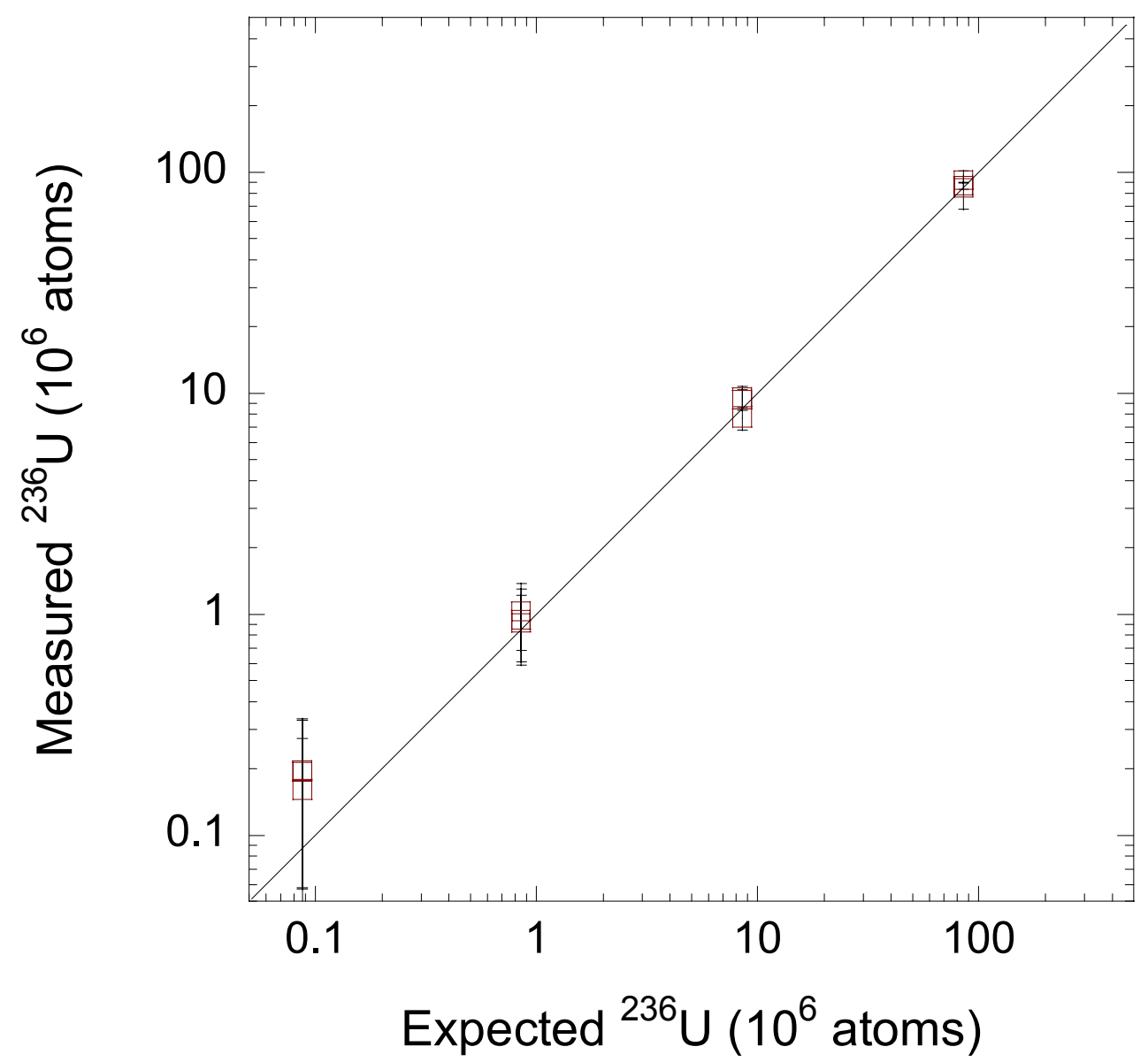

Fig. 3. 


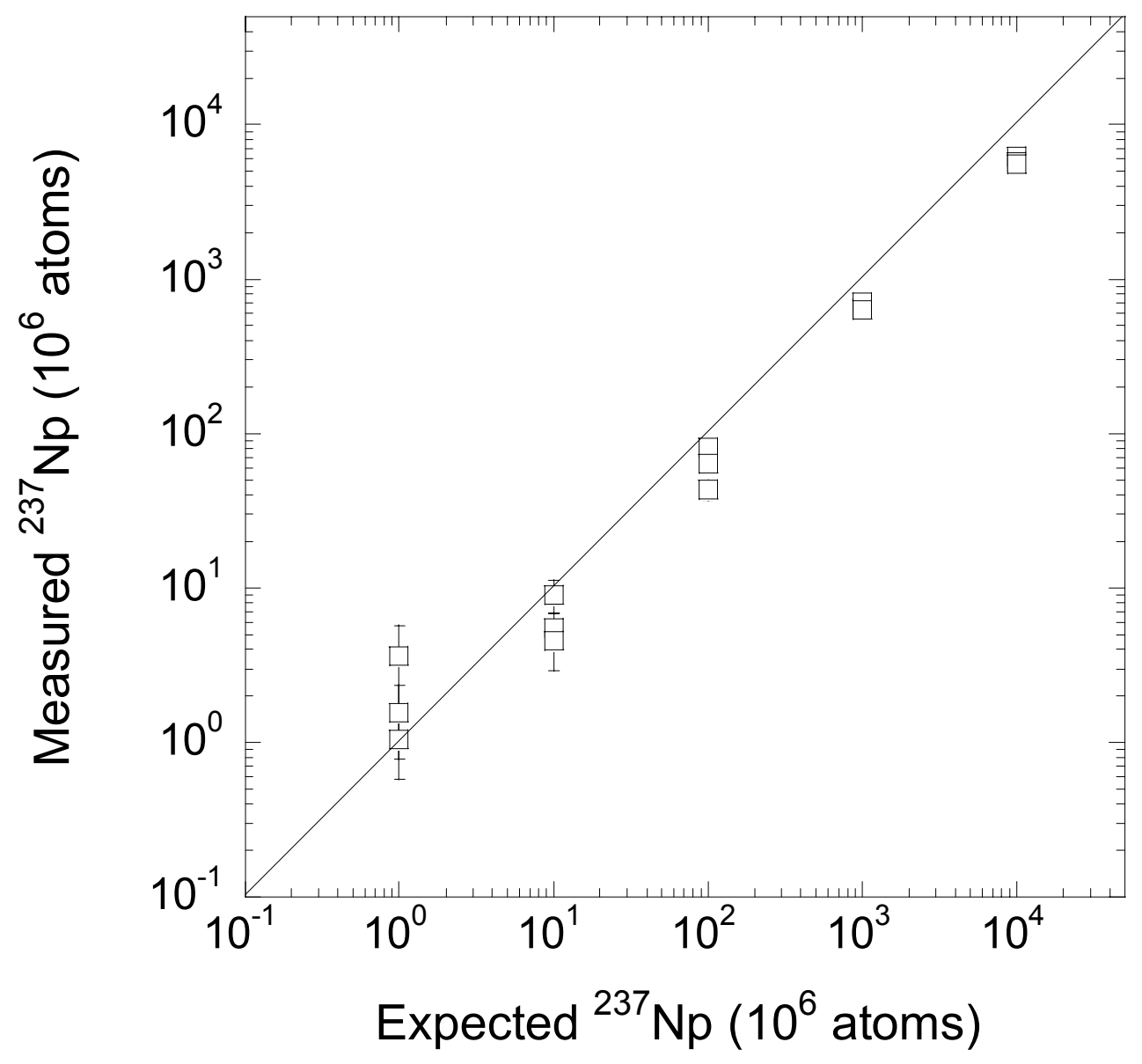

Fig. 4 